\title{
Phenytoin-Induced Gingival Overgrowth with Predominant Involvement of Hard Palate and Floor of Oral Cavity: A Case Report and Review of Literature
}

\author{
${ }^{1}$ Department of Neurosurgery, All India Institute of Medical \\ Sciences, New Delhi, India \\ 2Department of Pathology, All India Institute of Medical Sciences, \\ New Delhi, India \\ ${ }^{3}$ Department of Ear, Nose, and Throat and Head and Neck Surgery, \\ All India Institute of Medical Sciences, New Delhi, India
}

Rajeev Sharma ${ }^{1}$ Prasenjit Das ${ }^{2}$ Arvind Kairo ${ }^{3}$ Shashank S. Kale ${ }^{1}$

\begin{abstract}
Address for correspondence Rajeev Sharma, MCh, Department of Neurosurgery, All India Institute of Medical Sciences, Room 713, CNC, New Delhi, 110029, India (e-mail: rajufbd79@rediffmail.com).
\end{abstract}

J Neurosci Rural Pract 2020;11:349-352
Abstract
Keywords
- phenytoin-induced gingival overgrowth
- drug-induced gingival overgrowth
- drug-induced gingival enlargement
- drug-induced gingival hyperplasia
- drug-induced gingival hypertrophy

Drug-induced gingival overgrowth (DIGO) secondary to chronic phenytoin intake for seizure control is a well-recognized phenomenon. Phenytoin-induced gingival overgrowth (PIGO) usually resolves gradually following cessation of phenytoin intake. It is usually seen throughout the dentate regions of the maxillary and mandibular dental arches, but more severely affect their anterior portions exposed to atmosphere. We report a rare case of PIGO predominantly involving hard palate and floor of oral cavity, which has not been reported in English literature till date.

\section{Introduction}

Gingival overgrowth or enlargement is an esthetically disfiguring condition characterized by increased gingival connective tissue due to accumulation of extracellular matrix proteins in gingival fibroblasts in association with varying degrees of chronic inflammation. ${ }^{1}$ It is a very common complication of chronic phenytoin intake, usually seen in varying degrees of severity in nearly $85 \%$ patients, but is clinically significant in only approximately $50 \%$ patients. It occurs more commonly in children and youngsters than adults but affects both sexes equally. The enlargement though generalized throughout the dentate regions of oral cavity is nonuniform and remains confined to attached gingiva. It occurs maximally on the labial surfaces of the maxillary and mandibular anterior teeth. ${ }^{1}$ PIGO usually resolves spontaneously following cessation of drug intake. ${ }^{2}$

\section{Case Report}

A 21-year-old man presented with painless slowly progressive swellings in floor and roof of oral cavity since last 6 months. He was diagnosed as tectal plate glioma following seizures 18 years back and was on regular phenytoin therapy since then, currently at a dose of $300 \mathrm{mg}$ per day. His seizures were under control and tectal plate lesion was nonprogressive on serial neuroimaging. There was no history of bleeding from the oral swellings, and there was no family history of gingival overgrowth. His neurological examination was normal. Oral cavity examination showed two discrete areas of painless, pinkish, sessile, firm mucosal overgrowths involving floor of mouth $(4 \mathrm{~cm} \times 3 \mathrm{~cm}$ : black arrows $)$ and hard palate $(3 \mathrm{~cm} \times 2 \mathrm{~cm}$ : yellow arrows), with slight gingival overgrowths (comparatively much smaller: red arrows) over maxillary and mandibular alveolar arches ( - Fig. 1A). 


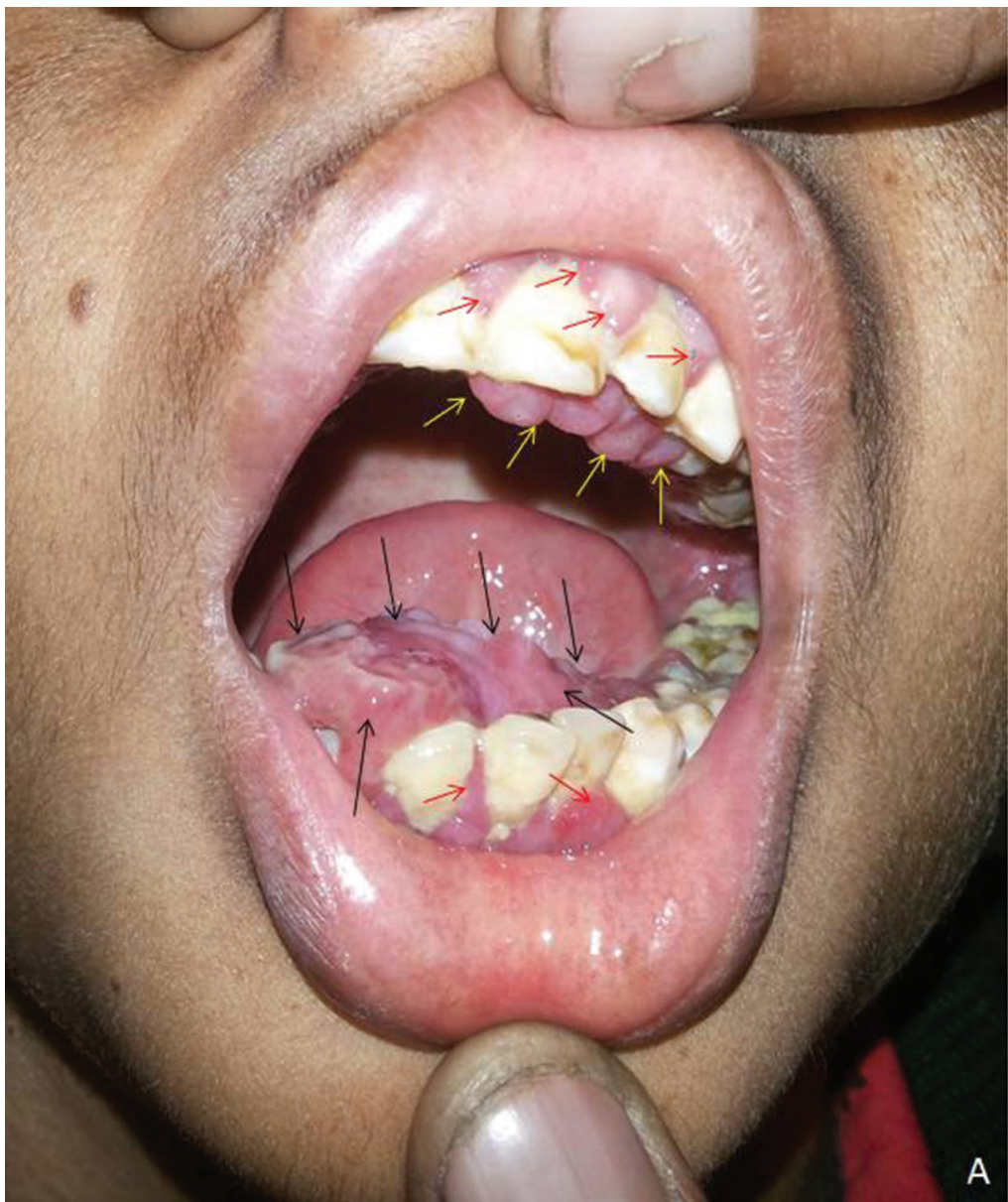

Day 1

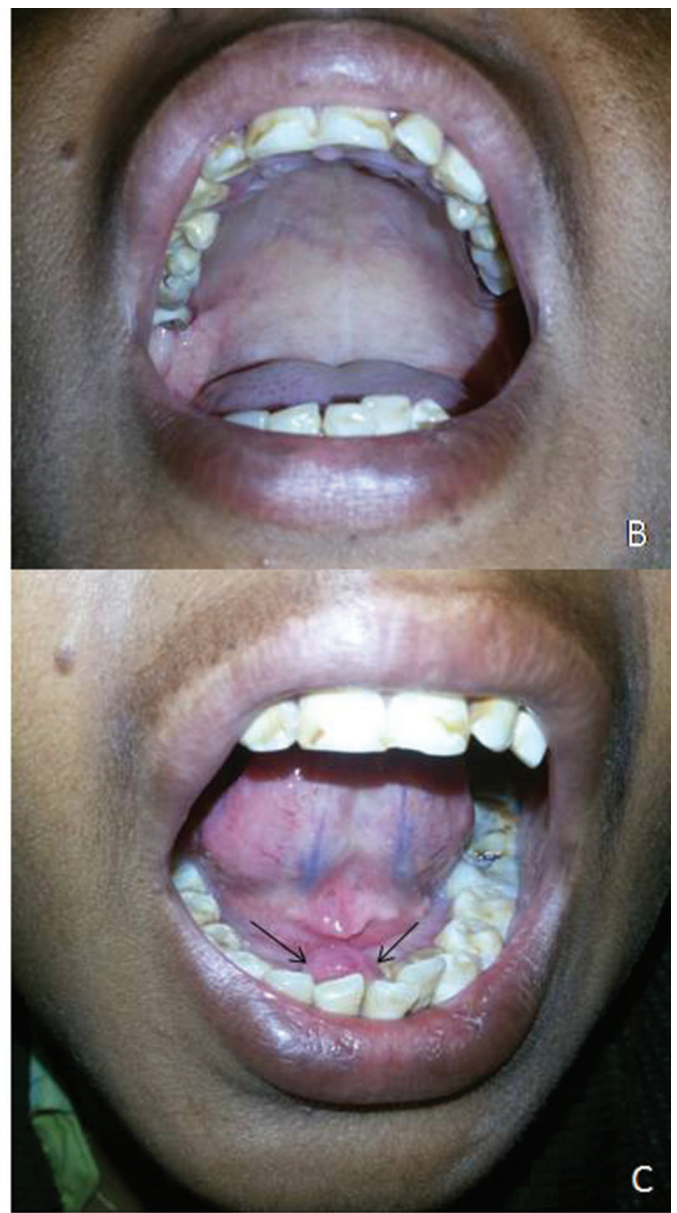

Day 77

Fig. 1 Oral cavity photographs showing (A) two pinkish sessile swellings involving hard palate and floor of oral cavity at day 1, (B) complete disappearance of palatal swelling, and (C) major reduction (>90\%) in size of oral cavity floor swelling at day 77 after phenytoin substitution with Levetiracetam

There was no bleeding on touching and probing the lesions. There were no skin or genital lesions and no mouth breathing on general examination. Provisional diagnosis of phenytoin-induced gingival overgrowth (PIGO) was made. Biopsy taken in view of atypical location of lesions was suggestive of gingival overgrowth (-Fig. 2 A-D), thereby confirming the diagnosis and ruling out neoplastic etiology. Phenytoin was replaced with levetiracetam as per weight, and oral hygiene improvement measures along with folic acid supplementation were started. Gradual reduction in size of palatal ( - Fig. 1B) and oral cavity floor ( - Fig. 1C) swellings was noted during serial follow-up, which further confirmed our diagnosis.

\section{Discussion}

This disfiguring adverse effect of Phenytoin was first reported by Kimball in 1939, within 1 year of introduction of phenytoin as an antiepileptic (1938). The fundamental disturbance occurs in certain genetically predetermined subpopulations of the gingival fibroblast. Various hypotheses like biochemical mechanistic unifying hypothesis proposed by Brown and Arany ${ }^{3}$ suggest it to be due to shift of collagen catabolism-anabolism balance to anabolic side. Gingival fibroblasts (responsible for gingival connective tissue turnover and remodelling) not only synthesize extracellular matrix structural proteins (especially collagen) but also degrade them, thereby maintaining homeostasis. Drug-plaque induced inflammation triggers matrix synthesis. High phenytoin concentration in gingival cells and gingival crevicular fluid reduces cation influx (mainly $\mathrm{Ca}^{2+}$ ) and interferes with active transport of folate through gingival fibroblasts cell membrane, thus reducing intracellular folate levels (local folic acid deficiency). This in turn reduces intracellular matrix metalloprotienases (MMP), E-cadherin, and SMAD synthesis. Matrix degradation is mediated by two tightly regulated pathways: extracellular enzymatic digestion (MMPs/TIMPs balance) and cellular phagocytosis. The matrix metalloproteinase (MMP, 1-28) enzyme family is primarily responsible for degradation of all structural proteins of extracellular matrix, and their activity is inhibited by an antagonistic group of tissue inhibitor of MMP (TIMP, 1-4). Phenytoininduced suppression of MMPs/TIMPs gene expression and reduced $\alpha 2 \beta 1$ integrin-mediated collagen endocytosis in gingival fibroblasts through MAPK (ERK1/2) and NF-кB 


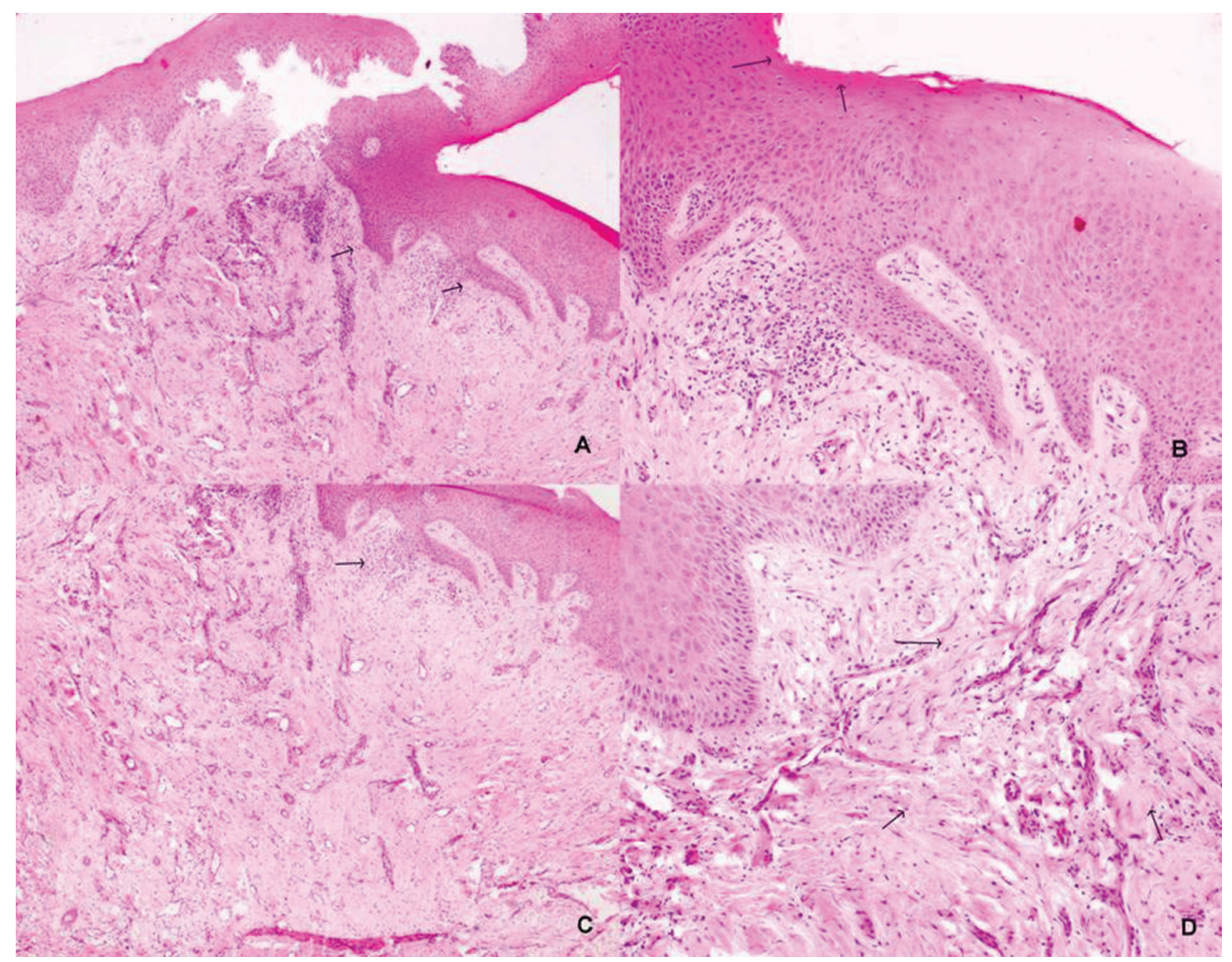

Fig. 2 (A) Biopsy from the lesion shows irregularly acanthotic stratified squamous epithelium with elongated irregular rete ridges (arrows; $\times 40$ ). (B) The mucosal epithelium in addition showed features of keratosis (arrows), without any dysplasia $(\times 100)$. (C) The subepithelium showed mild focal collection of lymphoid cells (arrow; $\times 40$ ) and (D) submucosal fibrosis (arrows; $\times 200$ ]. Plasma cells were sparse.

signaling pathways finally leads to accumulation of extracellular matrix. Also, no specific group of bacteria is associated with PIGO, ${ }^{3,4}$

The gingival enlargement usually starts as beadlike enlargement of the interdental gingiva and gradually progresses to involve marginal gingiva and form infection-prone pseudopockets, thereby initiating a vicious cycle. Extensive gingival enlargement may reach up to the crowns of teeth leading to chewing, swallowing, speech, and mouth closing difficulties besides aesthetic and psychological issues, compromised oral hygiene, bone destruction, and early teeth loss. ${ }^{1}$

Drug-induced gingival overgrowth is usually a clinical diagnosis and biopsy is not mandatory. ${ }^{4}$ Histopathological confirmation is mandatory in all gingival enlargements persisting or progressing despite adequate treatment. Histologically, there is a thickening of the squamous epithelium with a thin keratinous layer on its surface. Besides acanthosis and parakeratosis; there are elongated rete ridges/pegs, increased fibrosis, and chronic inflammatory cells in the lamina propria. Differential diagnosis includes chronic inflammatory enlargement like tuberculosis (usually painful), hereditary enlargement (more uniform involvement of oral cavity), neoplastic enlargement (focal, pedunculated, and bleeding), and secondary to systemic diseases like leukemia. Submucosal plasma cell granulomas and immunoglobulin (Ig)-G4 related diseases may also be considered in differential diagnoses, with characteristic plasma cell-rich infiltrate in the former and the storiform fibrosis with obliterative venulitis like features in the latter. ${ }^{5}$ Histopathological features in the present case lacked these findings.

Treatment options include plaque control measures, Phenytoin substitution, folic acid (topical and systemic) supplementation, azithromycin (topical and systemic), and surgical gingival recontouring procedures. Substitution of phenytoin with another effective antiepileptic drug (not causing PIGO) along with meticulous plaque control and folic acid supplementation is successful in many cases. It is imperative to continue this treatment and allow for 6 to 12 months to elapse between drug substitution and likely resolution of gingival overgrowth before declaring non-responders. Nonresponders may need multiple sessions 
of periodontal procedures like gingivoplasty, scalpel (internal bevel), or laser, assisted gingivectomy, or periodontal flaps. Prevention of this disfiguring complication by meticulous plaque control and folic acid supplementation together can improve drug compliance of this essential drug, thereby preventing life-threatening complications like break-through seizures. Predisposing factors include young age, poor oral hygiene, poor socioeconomic class, poor educational status, longer therapy duration, genetic predisposition, and polypharmacy. ${ }^{6,7}$

Signs and symptoms of gingival overgrowth are seen usually within 24 months of initiation of the medicine, and progresses more rapidly during first year of therapy. ${ }^{1}$ However, patient may be taking it since past many years but gingival overgrowth may be noted only recently since few months, ${ }^{8}$ as in our case. PIGO does not occur in edentulous areas and resolves spontaneously following dental extraction. The exclusive association of PIGO to anteriorly located dentate areas of the maxillary and mandibular alveolar arches in the oral cavity is well described in literature and can be explained by the prominent role of dental plaques in its etiopathogenesis. ${ }^{1}$ This is the first case report in English Literature documenting PIGO involving oral cavity floor and roof (palate) mucosa which responded well to plaque control, drug substitution, and folic acid supplementation. Dreyer and Thomas have reported a case of generalized palatal hyperplasia in an edentulous person on chronic phenytoin therapy. ${ }^{9}$

\section{Funding}

None.

\section{Conflict of Interest}

None declared.

\section{References}

1 Abdollahi M, Rahimi R, Radfar M. Current opinion on drug-induced oral reactions: a comprehensive review. J Contemp Dent Pract 2008;9(3):1-15

2 Marakoglu I, Gursoy UK, Cakmak H, Marakoglu K. Phenytoininduced gingival overgrowth in un-cooperated epilepsy patients. Yonsei Med J 2004;45(2):337-340

3 Brown RS, Arany PR. Mechanism of drug-induced gingival overgrowth revisited: a unifying hypothesis. Oral Dis 2015;21(1):e51-e61

4 Pette GA, Siegel MA, Parker WB. Gingival enlargement. J Am Dent Assoc 2011;142(11):1265-1268

5 Bharti V, Bansal C. Drug-induced gingival overgrowth: the nemesis of gingiva unravelled. J Indian Soc Periodontol 2013;17(2):182-187

6 Arya R, Gulati S. Phenytoin-induced gingival overgrowth. Acta Neurol Scand 2012;125(3):149-155

7 Thompson AL, Herman WW, Konzelman J, Collins MA. Treating patients with drug-induced gingival overgrowth. J Dent Hyg 2004;78(4):12

8 Agrawal AA. Gingival enlargements: Differential diagnosis and review of literature. World J Clin Cases 2015;3(9):779-788

9 Dreyer WP, Thomas CJ. Diphenylhydantoinate-induced hyperplasia of the masticatory mucosa in an edentulous epileptic patient. Oral Surg Oral Med Oral Pathol 1978;45(5):701-706 